\title{
Trend Following With Float-encoding Genetic Algorithm
}

\author{
Jiahua Luo \\ Department of Computer and Information Science \\ University of Macau \\ Av. Padre Tomas Pereira, Taipa, Macau \\ mb15457@umac.mo
}

\author{
Yain-Whar Si, Simon Fong \\ Department of Computer and Information Science \\ University of Macau \\ Av. Padre Tomas Pereira, Taipa, Macau \\ \{fstasp, ccfong\}@umac.mo
}

\begin{abstract}
Trend following plays an important role in technical analysis for trading financial instruments. In this paper, we propose a model based on Float-encoding Genetic Algorithm (FGA) to determine the best thresholds for trend following in financial time series. Trend following is based on the thresholds called $P \& Q$ which is calculated from the amount of an uptrend and downtrend to determine when to buy and sell at a particular time point. In our model, we first smooth the closing price by Exponential Moving Average (EMA) and partition the sample data into two parts respectively for training and testing. During the training session, FGA is used to find the best $P \& Q$ values which optimizes the average return based on a chosen EMA. The resulted $P \& Q$ is then evaluated against the testing data. Experiments conducted on Hang Sang Index (HSI) from Hong Kong shows promising results.
\end{abstract}

Keywords-Stock trend following; P\&Q; Float-encoding Genetic Algorithm; Exponential Moving Average

\section{INTRODUCTION}

There are two approaches for trading in financial markets; fundamental analysis and technical analysis. In fundamental analysis, the movement of the price is predicted based on the factors such as profit and loss, performance data, and analysis for the corresponding industry [11]. Other factors such as government policies and political climate are also taken into account for the analysis. Fundamental analysis often fails to predict price movement due to the sheer number of factors involved and the influence of the biases from the analysts' judgment.

In technical analysis, factors such as historical price, volume, and other statistical data are used to predict the future price movement [10]. In technical analysis it is assumed that all information is already reflected in the price data [11] and charts and other tools are used to identify patterns that can suggest future activity. A number of technical indicators can be included in technical analysis such as MA, EMA, MACD, and RSI.

One of the strategies used in technical analysis is called trend following [12] in which trading decisions are made based on the measure of price increase or decrease from a chosen time point. In trend following, predefined rules are often used to monitor the market trend and traders follow the direction of the market in making decision. For instance, when the price goes up, it is likely that the uptrend will continue for a certain period of time and therefore the trader may take a long position. When the price drops, it is often the case that the price will further decrease for a certain period of time and therefore trader may start selling. Such buying and selling decisions are made when the uptrend or downtrend is greater than predefined thresholds. A number of approaches for calculating the thresholds have been reported in literature. In this paper, we propose a model based on Floatencoding Genetic Algorithm (FGA) to determine the best thresholds for trend following in financial time series.

We introduce the definition of thresholds and the procedure of Float-coding Genetic Algorithm in section 2. The results of the experiment on HSI data is detailed in section 3. Related work is reviewed in section 4 and we conclude the paper with future work in section 5 .

\section{TRANSACTION MODEL IN STOCK TREND FOLLOWING}

When to buy and sell is one of the key problems in the trend following. Therefore, a pair of threshold values $P \& Q$ is defined in [7] to denote the amount of an uptrend (downtrend) that triggers a buying (selling) signal. The process of $P \& Q$ in generating trading signals is depicted in Figure 1. In trend following, a buy signal is generated when the uptrend in price is greater than a predefined threshold $P$. A sell signal is generated when the downtrend exceeds a predefined threshold $Q$.

Two methods of determining $P \& Q$ are discussed in [7]. They can be classified as static or adaptive approaches where in the former it remains constant over the whole stock periods and in the latter, it changes to adapt different trends. However, these methods rely on heuristics to determine the values P\&Q. In this paper, we propose an approach for determining the value of $P \& Q$ based on Float-encoding Genetic Algorithm (FGA). In our approach, user provides the ranges for $P \& Q$ based on the desired level of risk. Based on these ranges, our proposed algorithm calculates the values of $P$ and $Q$ that maximize the average return over the last 10 years. 


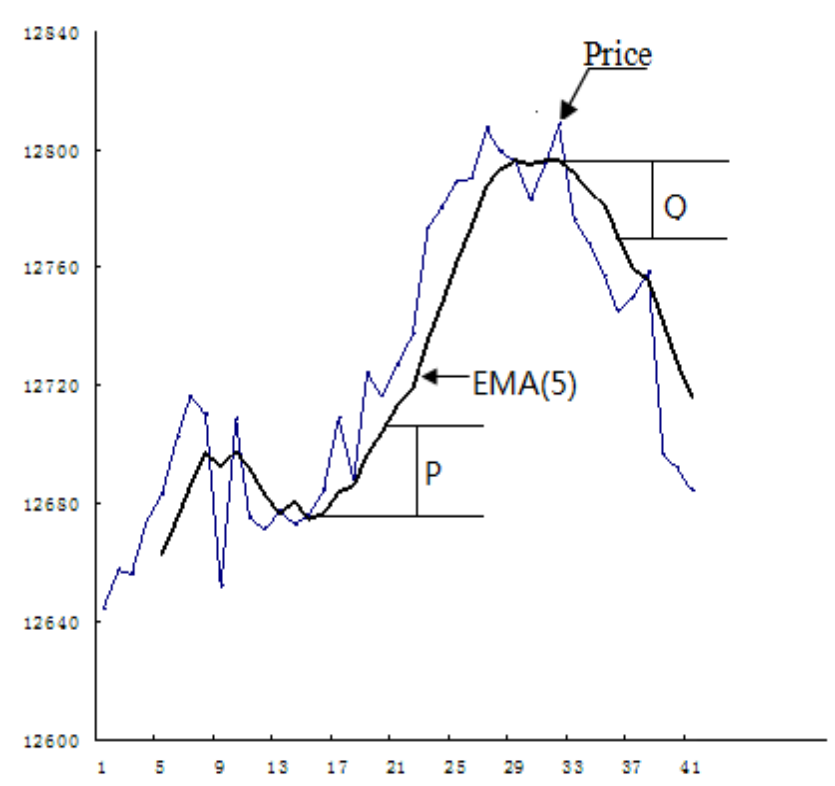

Figure 1. P\&Q in trend following based on $\operatorname{EMA(5).~}$

Fluctuation occurs in stock price. In order to apply $P \& Q$, it is necessary to smooth the fluctuated price trend through technical indicators such as Simple Moving Average (SMA), Weighted Moving Average (WMA), Exponential Moving Average (EMA), etc. In this paper, we adopt EMA which gives more weight to recent prices and ever-decreasing weight to older data relative to the SMA and WMA. EMA can be calculated as follows:

$$
E M A(t)=(\operatorname{price}(t)-\operatorname{EMA}(t-1)) \times \frac{2}{n+1}+\operatorname{EMA}(t-1)
$$

where $t$ and $n$ are the transaction day and smoothing day. Different value of $n$ can greatly affect the result in our model. price $(t)$ is the closing price on $t^{\text {th }}$ day.

In this paper, we use a Genetic Algorithm (FGA) to find the values of $P \& Q$ that optimize the average return. GA uses stochastic operators instead of deterministic rules to search the solution space and it employs implicit parallelism. However, the binary representation for defining chromosomes is unsuitable for multidimensional or large range numerical problems. For example, in our model, the ranges provided by the user for $P$ and $Q$ can be large depending on the acceptable risk level and therefore the corresponding chromosome with binary encoding can be very long. To alleviate this problem, the Float-encoding Genetic Algorithm is used in this paper. Unlike the binary encoding, FGA uses real value as the genes of chromosome together with special operators. As in the binary encoding GA, there are three basic operators in the FGA [1]: selection, crossover and mutation. In every generation, the fitness of each individual is calculated. Individuals with higher degree of fitness are allowed to survive in the next generation, while the weaker ones are replaced. We define the chromosome as a pair of floating point values representing $P \& Q$.

The fitness function for the proposed FGA is defined in terms of average return resulted from using the corresponding $P \& Q$ pair in the chromosome. Average return is calculated based on the daily closing price. For the sake of simplification, we ignore the dividend and other service charges in calculation. We can define the average return as follows:

$$
A v g_{-} \text {return }=\frac{1}{N} \sum_{i=1}^{N}\left(\frac{\operatorname{sell}\left(i, t^{\prime}\right)-\operatorname{buy}(i, t)}{\operatorname{buy}(i, t)}\right)
$$

where $\operatorname{buy}(i, t)$ and $\operatorname{sell}\left(i, t^{\prime}\right)$ are the buying price and selling price in the $i^{\text {th }}$ transaction. A transaction comprises a buying and its subsequent selling actions. $t$ and $t$ ' are the timestamps of this two actions respectively. $N$ is the total pair of transactions in the training data.

The purpose of selection operator is to select the individuals which have higher degree of fitness for evolution. The selected individuals are then stored in the "mating pool" based on their fitness values. In this paper, we adopt Roulette Wheel Selection method and the selection operator proposed in [1] is adopted for our model.

1) Calculate the fitness value fitness $\left(v_{i}\right)$ for each individual $v_{i}(i=1, \ldots, Z)$, where $Z$ is the total number of individuals in the population.

2) Calculate the proportion of each individual

$$
\operatorname{prop}\left(v_{i}\right)=\frac{\operatorname{fitness}\left(v_{i}\right)}{\sum_{j=1}^{Z} \operatorname{fitness}\left(v_{j}\right)}
$$

3) Calculate the selection probability of each individual

$$
P\left(v_{i}\right)=\sum_{j=1}^{i} \operatorname{prop}\left(v_{j}\right)
$$

The selection probability of $i^{\text {th }}$ individual is the defined as the cumulated proportion from the first individual to the $i^{\text {th }}$ individual. Next, a random number $R(0 \leq R \leq 1)$ is generated. Based on $R$, we select the $(i+1)^{\text {th }}$ individual that meets the condition $P\left(v_{i}\right)<R<P\left(v_{i+1}\right)$. Repeat this step until the required number of individuals is selected.

Crossover is a crucial operator in the FGA. During the evolution, crossover combines genes to generate new solutions. Crossover operator can be defined as follows:

$$
\begin{aligned}
& \text { begin } \\
& \qquad \begin{aligned}
& P_{1}^{\prime}:=(1-\alpha) \cdot P_{1}+\beta \cdot P_{2} \\
& P_{2}^{\prime}:=(1-\beta) \cdot P_{2}+\alpha \cdot P_{1} ; \\
& \text { for } \mathrm{i}=1 \text { to } 2 \text { do } \\
& \\
& \quad \text { if } P_{i}^{\prime}<L \text { then } P_{i}^{\prime}:=L ; \\
& \quad \text { if } P_{i}^{\prime}>R \text { then } P_{i}^{\prime}:=R ;
\end{aligned} \\
& \text { end }
\end{aligned}
$$

where $P_{1}$ and $P_{2}$ are the genes of two parents respectively. $P_{1}^{\prime}$ and $P_{2}^{\prime}$ are the corresponding new genes of offspring after the crossover operation. $\alpha$ and $\beta$ are the random numbers in the range $(0, r)$ where $r<=1$. $L$ and $R$ are the lower and upper bounds for $P$ value. Crossover operation for $Q$ is also defined in a similar way.

Mutation is used to alter one or more gene values in a chromosome from its current state. Mutation reduces the possibility of the searching process falling into local optimal solution. The mutation operator is defined as follows: 


$$
P^{\prime}=\left\{\begin{array}{l}
P+k \cdot(R-P) \cdot \gamma, \text { when rand }=0 \\
P-k \cdot(P-L) \cdot \gamma, \text { when rand }=1
\end{array}\right.
$$

where $\gamma$ is a random number in the range of zero and one. $k$ is a constant learning parameter. $P^{\prime}$ and $P$ are the genes of new and old chromosome. $L$ and $R$ are the lower and upper bounds for $P$ and they are predefined before the mutation operation. The purpose of the function rand is to generate either 0 or 1 . Mutation operation for $Q$ can be also defined in a similar way.

\section{EXPERIMENTAL RESULTS}

Experiments are conducted on the historical close price of the inter-day data during the period from 01-01-2001 to 14-092011 of Hang Seng Index (HSI) from Hong Kong Stock Market. From this period, we use the first 2000 records (from 01-012001 to 19-01-2009) for training, and the rest of the records (from 20-01-2009 to 14-09-2011) are used for testing. EMA(5) is also calculated on the stock data to smooth out the fluctuation in the original price. The HSI and its EMA values are depicted in Figure 2.

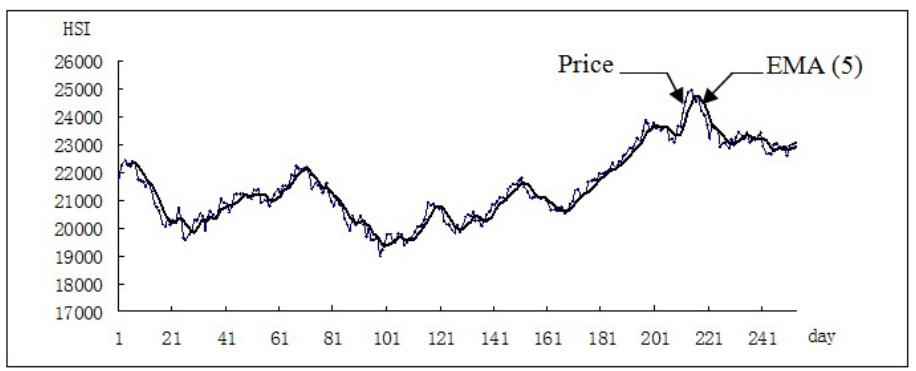

Figure 2. Closing price and its 5-day EMA value of HSI in 2010.

Following parameters are used for FGA in our experiment:

- $\quad$ The total number of generations $=3000$.

- $\quad$ The size of population in each generation $=100$.

- The proportion of individuals kept for the next generation $=30 \%$. These individuals are the fittest members from the entire generation.

- $\quad$ The probability for the crossover operation $=0.8$. (i.e. a randomly selected pair of chromosomes has the 0.8 probability to generate new offspring by crossover operator.)

- $\quad$ The probability for the mutation operation $=0.1$. (i.e. the genes of a chromosome have 0.1 probability for being mutated.)

Before the calculation, the ranges for $P$ and $Q$ can be input by the user based on their trading strategies and risk appetite. Next, FGA finds the best $P \& Q$ values which optimizes the average return for the given training period. Average return is calculated based on the daily close price from the historical data. Then the found $P \& Q$ values are used for trading on the testing period. Finally, the average return made during the testing period is recorded for analysis.

In Table 1 we show the results corresponding to different predefined $P \& Q$ ranges and EMA parameters. The EMA parameters considered in our model are 5, 10, 21, 30 and 50 which are widely used for smoothing price data in technical analysis. Note that the ranges predefined for $P \& Q$ can implicitly reflect the risk appetite of the trader. Small ranges for $P \& Q$ can trigger more trading signals although some of them are unprofitable. We compile a subset of cases in Table 1 for some combination of $P \& Q$ and EMA. TTR and TTS denote the total number of transaction signals during training and testing intervals. Return1 and Return2 denote the net average return during training and testing intervals.

From the Table 1, we can observe that larger the parameter of EMA, the less trading signals are generated. The numbers of generated signals are relatively lower for larger EMA since the trends produced by EMA are smoother compared to smaller EMA. In addition, we can observe that the returns generated in testing period (the rightmost column of Table 1) for smaller EMA are lower than the returns generated for larger EMA indicating the presence of false trading signals.

Using small ranges for $P$ or $Q$ and a short-day EMA can trigger high volume of false trading signals and thus cause the so-called high-frequency trading. In contrast, larger ranges for $P$ or $Q$ and a long-day EMA can also overlook significant number of suitable trading signals. The experimental results given in Table 1 highlight the fact that the choice of $P \& Q$ and EMA can significantly influence the outcome of the trend following.

TABLE 1. TESTING RESUlt BASED ON DIFFERENT RANGES AND EMA VALUES.

\begin{tabular}{|c|c|c|c|c|c|c|c|c|}
\hline Range of $P$ & Range of $Q$ & EMA & $P$ & $\mathbf{Q}$ & TTR & Return1 & TTS & Return2 \\
\hline \multirow{5}{*}[0,300]{} & \multirow{5}{*}[0,300]{} & 5 & 154.57 & 185.59 & 69 & $0.7 \%$ & 16 & $2.5 \%$ \\
\hline & & 10 & 271.66 & 263.01 & 23 & $4.3 \%$ & 10 & $2.9 \%$ \\
\hline & & 21 & 169.75 & 217.92 & 18 & $5.3 \%$ & 4 & $15 \%$ \\
\hline & & 30 & 273.20 & 57.03 & 16 & $4.8 \%$ & 6 & $2.3 \%$ \\
\hline & & 50 & 228.55 & 273.16 & 12 & $6.3 \%$ & 2 & $15.1 \%$ \\
\hline \multirow{5}{*}[0,600]{} & \multirow{5}{*}[0,600]{} & 5 & 535.93 & 597.44 & 21 & $4.7 \%$ & 11 & $0.6 \%$ \\
\hline & & 10 & 486.26 & 456.93 & 17 & $6.1 \%$ & 8 & $2.6 \%$ \\
\hline & & 21 & 169.48 & 521.25 & 14 & $5.9 \%$ & 3 & $15.9 \%$ \\
\hline & & 30 & 586.19 & 545.36 & 8 & $9.2 \%$ & 2 & $17.0 \%$ \\
\hline & & 50 & 361.27 & 473.91 & 6 & $17.9 \%$ & 2 & $12.0 \%$ \\
\hline \multirow{5}{*}[0,1000]{} & \multirow{5}{*}[0,1000]{} & 5 & 767.62 & 441.73 & 18 & $6.0 \%$ & 10 & $0.4 \%$ \\
\hline & & 10 & 485.17 & 455.41 & 17 & $6.1 \%$ & 8 & $2.6 \%$ \\
\hline & & 21 & 746.42 & 841.12 & 5 & $11.2 \%$ & 2 & $21.8 \%$ \\
\hline & & 30 & 607.65 & 785.78 & 5 & $24.4 \%$ & 2 & $14.7 \%$ \\
\hline & & 50 & 984.85 & 488.12 & 5 & $19.3 \%$ & 1 & $36.1 \%$ \\
\hline
\end{tabular}




\section{RELATED WORK}

In algorithmic trading, trading operations can be wholly or partially executed automatically by the computers [2]. Artificial intelligence is one of the core methods to make strategies in the automatic trading. A number of recent work have been reported on automatic trading using evolutionary computation (e.g. [3][4][6]). Trend following is an important strategy in the algorithmic trading which follows the stock trend and then make trading decisions. However, mistaken beliefs, psychological factors, and biases can cause the wrong parameters to be selected for trend following. Effect by behavioral biases on technical trend followers' trading performance is studied via a simulation based on moving average strategy in [5]. Thus, scientifically determining the time for buying and selling is essential.

Trend following can probably accumulate profit in the long run by obtaining more gains than loses. However, it doesn't guarantee profit every time and it poorly performs especially during the period when certain form of fluctuation occurs. Some scholars have applied different strategies to determine the trading signals by dynamically adapting to the fluctuation in the stock trend. For instance, Fong et al. adopted RSI (Relative Strengthen Index, a technical analysis indicator) to fit different trends [7] and a Trend Recalling model is proposed to match the current trend with the past underlying historic data to recognize the financial cycle [8].

\section{CONCLUSION AND FUTURE WORK}

In this paper, we introduce an evolutionary approach for trend following based on Float-encoding Genetic Algorithm. FGA in our model uses implicit parallel search to find the best $P \& Q$ values for optimizing the returns. The proposed model was trained and tested on the historical price data from Hong Kong Hang Sang Index. The experimental results show that proposed trend following with FGA approach achieves promising result in inter-day trading sessions.

We also observe that the model achieves relatively high returns in the stock with high fluctuations and performs poorly in the stock with relatively stable fluctuation. We also find that the model often generates false trading signals while tracking short uptrends or downtrends. This is due to the fact that only one pair of $P \& Q$ is used throughout the training and testing period and the chosen $P \& Q$ is not completely suitable to the fluctuation in different period of the stock. In order to alleviate this problem, we are planning to segment the stock trend data into different fluctuation intervals before using FGA to determine the optimal $P \& Q$.

\section{REFERENCES}

[1] C. T. Su, W. T. Tyen, "A Genetic Algorithm Approach Employing Floating Point Representation for Economic Dispatch of Electric Power Systems," International Congress on Modeling and Simulation, pp. 14441449, 1997.

[2] G. Nuti, M. Mirghaem, P.C. Treleaven, and C. Yingsaeree, Algorithmic Trading, IEEE Computer, pp.61-69, November, 2011.

[3] A. Brabazon, M. O'Neill, I. Dempsey, “An introduction to Evolutionary Computation in Finance," IEEE Computational Intelligence Magazine, pp.43-55, November, 2008.

[4] P. Fernández-Blanco, D. Bodas-Sagi, J. F. Soltero, and I. J. Hidalgo, "Technical Market Indicators Optimization using Evolutionary Algorithms," Proceedings of the 2008 GECCO conference companion on Genetic and evolutionary computation (GECCO '08), Maarten Keijzer (Ed.), pp. 1851-1857, ACM, 2008.

[5] H. Cheung, K. K. Lai, K. Liu, "Performance of Biased Trend-following Stock Investor in Mainland China and Hong Kong," Fourth International Joint Conference on Computational Sciences and Optimization, pp. 548552, IEEE, 2011.

[6] K. Matsui, H. Sato, "Neighborhood Evaluation in Acquiring Stock Trading Strategy Using Genetic Algorithms," International Conference of Soft Computing and Pattern Recognition, pp. 369-372, 2010.

[7] S. Fong, J. Tai, Y. W. Si, "Trend Following Algorithms for Technical Trading in Stock Market," Journal of Emerging Technologies in Web Intelligence (JETWI), 3(2), pp. 136-145, May, 2011.

[8] S. Fong, J. Tai, "Improved Trend Following Trading Model by Recalling Past Strategies in Derivatives Market," The Third International Conferences on Pervasive Patterns and Applications, pp. 32-36, 2011.

[9] http://finance.yahoo.com/

[10] Investopedia, Technical Analysis, http://www.investopedia.com/terms/t/technicalanalysis.asp

[11] Wikipedia, Fundamental Analysis, http://en.wikipedia.org/wiki/Fundamental_analysis

[12] S. Fong, J. Tai, "The Application of Trend Following Strategies in Stock Market Trading," The 5th International Conference on Networked Computing, pp. 1971-1976, 2009. 